\title{
Thermoelastic Stress Analysis: An NDE Tool for the Residual Stress Assessment of Metallic Alloys
}

During manufacturing, certain propulsion components that will be used in a cyclic fatigue environment are fabricated to contain compressive residual stresses on their surfaces because these stresses inhibit the nucleation of cracks. Overloads and elevatedtemperature excursions cause the induced residual stresses to dissipate while the component is still in service, lowering its resistance to crack initiation. Research at the NASA Glenn Research Center at Lewis Field has focused on employing the Thermoelastic Stress Analysis technique (TSA, also recognized as SPATE: Stress Pattern Analysis by Thermal Emission) as a tool for monitoring the residual stress state of propulsion components.

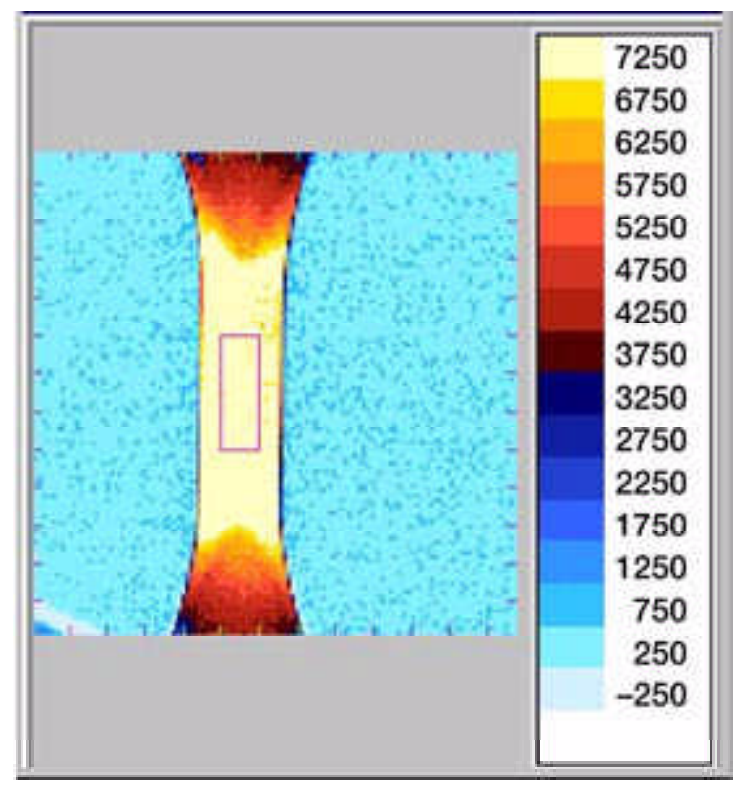

Typical infrared test image for a dogbone TIMETAL $21 S$ specimen. The rectangular box within the specimen indicates the area where the average signal was obtained. The scale displays the dimensionless digital values of the infrared camera signal corresponding to the cyclic temperature amplitude.

TSA is based on the fact that materials experience small temperature changes when they are compressed or expanded. When a structure is cyclically loaded (i.e., cyclically compressed and expanded), the resulting surface-temperature profile correlates to the stress state of the structure's surface. The surface-temperature variations resulting from a cyclic load are measured with an infrared camera. Traditionally, the temperature amplitude of a TSA signal has been theoretically defined to be linearly dependent on the cyclic stress amplitude. As a result, the temperature amplitude resulting from an applied cyclic stress 
was assumed to be independent of the cyclic mean stress.

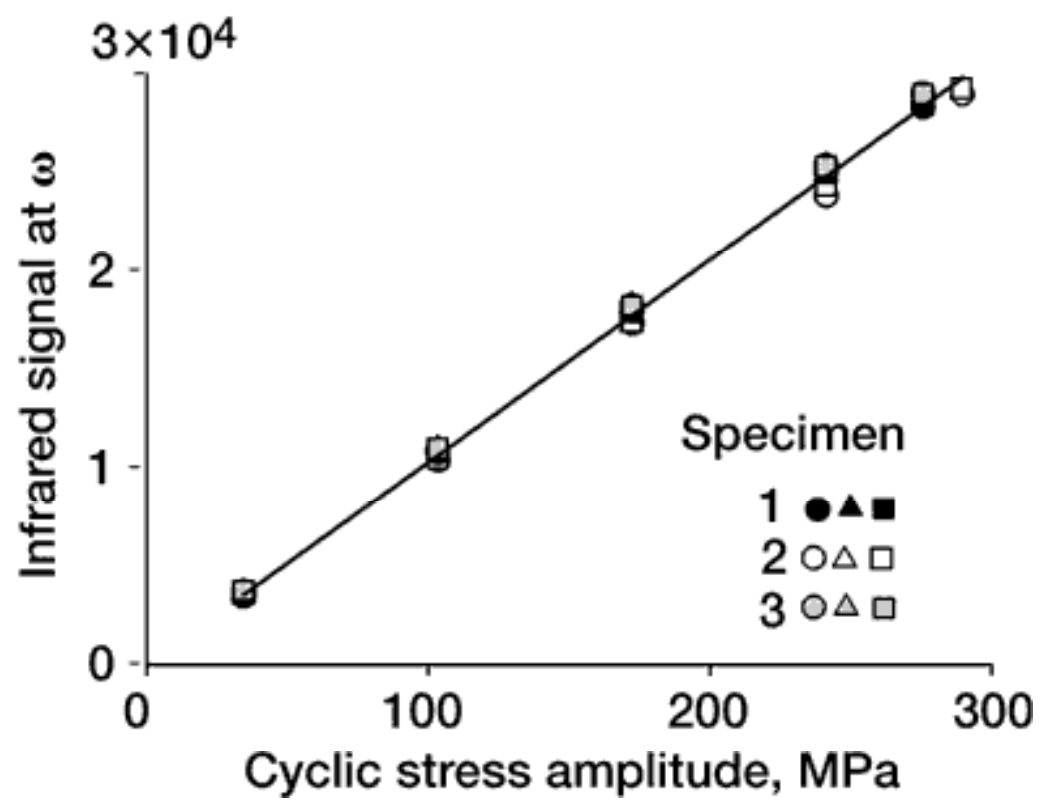

First harmonic $(\omega)$ infrared signal output at various stress amplitudes $\left(\sigma_{m}=0\right)$ for TIMETAL21S. Indicated is the linear relationship of the TSA signal to the cyclic stress amplitude for a fixed mean stress. Mean stress variations would affect the data by changing the slope of the line.

Recent studies established that the temperature response also depends on the cyclic mean stress or static stress. A study by the authors (ref. 1) showed that static stresses significantly influenced the TSA results for titanium- and nickel-based alloys. A more indepth analysis, which involved analyzing multiple harmonics of the temperature response, was also conducted (ref. 2). This research showed that the thermoelastic response of a structure subjected to a pure sinusoidal mechanical load with frequency $\omega$ produced a TSA signal with frequency components at the primary frequency, $\omega$, as well as at the second harmonic, $2 \omega$. The first harmonic of the thermal response is a function of the cyclic stress amplitude and the mean stress, whereas the second harmonic is a function of the square of the stress amplitude. By obtaining the TSA amplitudes of the first and second harmonics, we are now able to obtain the stress amplitude and the mean stress at a given point on a structure subjected to a cyclic load simultaneously. The rather complex analysis of the temperature response involved obtaining the first and second harmonic amplitudes for 16,384 infrared detectors (in a 128 by 128 focal plane array). In addition, comparisons were made between the experimental data and theoretical predictions that were based on a revised theory that takes into account the mean stress effect. Good agreement was achieved. 


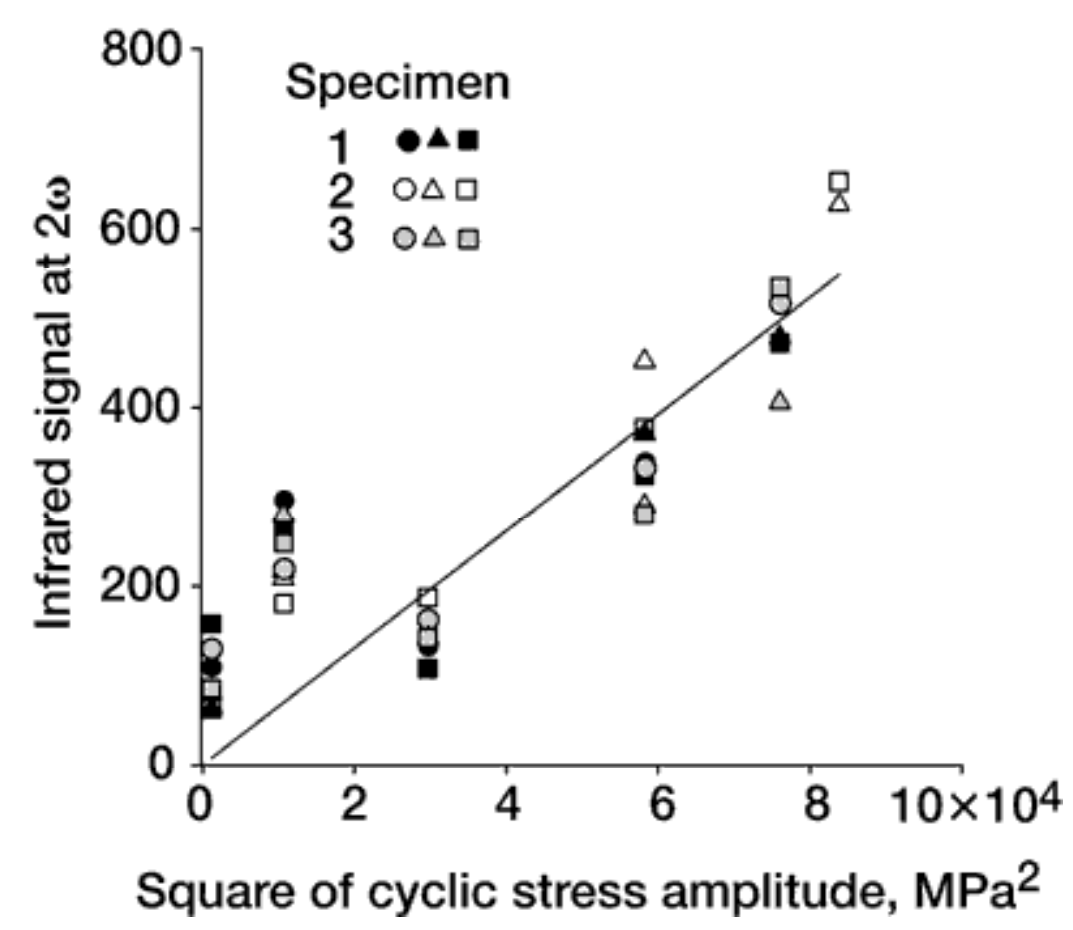

Second harmonic $(2 \omega)$ infrared signal output at various stress amplitudes $\left(\sigma_{m}=0\right)$. Indicated is the linear relationship of the TSA signal to the square of the cyclic stress amplitude. The second harmonic TSA response is independent of the cyclic mean stresses.

Since confidence was achieved concerning reliable TSA measurements of the mean stress effect (i.e., the static stress), research can focus on the application of the method to residual stress assessment. Such measurements will assist researchers in the characterization of materials in the laboratory as well as in the in situ monitoring of the current residual stress state in actual structural components during fabrication and service.

\section{References}

1. Gyekenyesi, A.L.; and Baaklini G.Y.: Thermoelastic Stress Analysis: The Mean Stress Effect in Metallic Alloys. NASA/TM—1999-209376, 1999.

2. Gyekenyesi, A.L.; and Baaklini G.Y.: Quantifying Residual Stresses by Means of Thermoelastic Stress Analysis. SPIE: Conference on the NDE of Aging Materials and Composites, Newport Beach, CA, March 5-9, 2000.

Ohio Aerospace Institute contact: Dr. Andrew L. Gyekenyesi, (216) 433-8155, Andrew.L.Gyekenyesi@grc.nasa.gov

Glenn contact: Dr. George Y. Baaklini, (216) 433-6016, George.Y.Baaklini@grc.nasa.gov

Authors: Dr. Andrew L. Gyekenyesi and Dr. George Y. Baaklini 
Headquarters program office: OAST

Program/Project: AvSP 